\title{
Finiteness and choice
}

by

\author{
Omar De la Cruz (West Lafayette, IN)
}

\begin{abstract}
We deal with weak choice principles of the form: Every "finite" family of non-empty sets has a choice function, where "finite" stands for one of several different definitions of finiteness that are not equivalent unless we assume the axiom of choice (AC). Several relations of implication and independence are established. In the process, we answer a few open questions about the relations between different definitions of finiteness.
\end{abstract}

1. Introduction. The following is a well known result in set theory without the axiom of choice:

\section{Every finite family of non-empty sets has a choice function.}

(see Section 3.1 for the definition of choice function). The axiom of choice (AC) states that this also holds for infinite families. However, without AC, the notion of finiteness itself is not so clear, since different statements that express properties that we expect finite sets to have and which are equivalent under AC, are not provably equivalent without the axiom.

From the several statements considered in the literature as possible definitions of finiteness, in particular in Tarski [Tar24], Lévy [Lév58], and Spišiak and Vojtáś [SV88], as well as in Howard and Rubin [HR98], it is commonly agreed that the "right" definition is the most restrictive one: namely, equivalence with a natural number (see notion I below, as well as Remark 1.2). It is this notion of finiteness for which (1) is true.

In this article we study the relative strength of principles obtained by modifying (1) to use less restrictive notions of finiteness, and more principles that are obtained from these by weakening the definition of choice function. It happens that none of these modifications of (1) can be proved without assuming at least a weak form of AC. Many easy relations can be found between these statements and between these and some other well known

2000 Mathematics Subject Classification: 03E30, 03E25, 03E35.

This article is based on a chapter of the author's doctoral dissertation. The author would like to thank his thesis advisor, William J. Mitchell, for his guidance and help. 
choice principles; other not so trivial relations are established in this article. It turns out that these interrelations are different for ZF, the ZermeloFraenkel axioms for set theory, and for ZFA, a weakened version of ZF in which the axiom of extensionality is modified in order to allow the existence of atoms. For a more complete exposition and complete lists of axioms, see for example Jech [Jec73]. Notice in particular that every theorem of ZFA is also a theorem of ZF; unless otherwise stated, all results here can be obtained from the weaker theory ZFA.

In Section 2 we list the notions of finiteness to be used and establish all the relationships between them (for which we answer a few open questions). These results are summarized in Figure 1. In Section 3 we state the choice principles we will consider, and we find several implications, while in Section 4 we establish some independence results about them. Our results are summarized in Figure 2.

2. Notions of finiteness. The first group of notions is taken from Lévy [Lév58], who established all the relations of implication and independence (with respect to ZFA) between them. Lévy introduced Ia and VII, while definitions I, II, III, and V were introduced by Tarski [Tar24], as well as VI (attributed to Tarski by Mostowski [Mos38]). Notion IV was originally introduced by Dedekind.

Definition 1. A set $X$ is said to be:

1. I-finite if every non-empty family of subsets of $X$ has a maximal (or minimal) element under inclusion.

2. Ia-finite if $X$ is not the disjoint union of two non-I-finite sets.

3. II-finite if every non-empty family of subsets of $X$ which is linearly ordered by inclusion has a maximal (or minimal) element under inclusion.

4. III-finite if there is no one-to-one map from $\mathcal{P}(X)$ into a proper subset of $\mathcal{P}(X)$.

5. IV-finite (also Dedekind finite) if there is no one-to-one map from $X$ into a proper subset of $X$.

6. V-finite if $X=\emptyset$ or there is no one-to-one map from $2 \times X$ into $X$.

7. VI-finite if either $X$ is empty, or it is a singleton, or there is no one-to-one map from $X \times X$ into $X$.

8. VII-finite if $X$ is I-finite or it is not well orderable.

REmarks 1. 1. To each notion of finiteness $\mathcal{Q}$ corresponds a notion of infinity: we will say that a set is $\mathcal{Q}$-infinite if it is not $\mathcal{Q}$-finite.

2. A set $X$ is I-finite if and only if there is a bijection between $X$ and an ordinal $n<\omega$ (see Tarski [Tar24]). This one is the commonly accepted definition of finiteness, among other reasons, because it is absolute for models 
of ZF. Therefore, whenever we use the terms finite and infinite without further qualification, we mean I-finite and I-infinite, respectively.

3. An infinite set $X$ which is Ia-finite is also called amorphous. An Iainfinite set is also called partible.

4. A set $X$ is IV-infinite if and only if it contains a well orderable infinite subset. A set $X$ is III-infinite if and only if $\mathcal{P}(X)$ is IV-infinite, and if and only if it has a well ordered infinite partition.

Cardinal numbers, as well as operations and order on cardinal numbers, can be defined without using AC (see, for example, Jech [Jec73]). Using those definitions we can rewrite some of the definitions in the following way:

1. $X$ is I-finite iff $|X|<\aleph_{0}$.

2. $X$ is III-finite iff $2^{|X|}+1>2^{|X|}$.

3. $X$ is IV-finite iff $|X|+1>|X|$ iff $|X| \nsupseteq \aleph_{0}$.

4. $X$ is $\mathrm{V}$-finite iff $|X|=0$ or $2 \cdot|X|>|X|$.

5. $X$ is VI-finite iff $|X|=0,1$ or $|X|^{2}>|X|$.

Spišiak and Vojtáš [SV88] introduced the following way to derive new definitions of finiteness from old ones.

Definition 2. Let $\mathcal{Q}$ be any notion of finiteness. We say that a set $X$ is $\mathcal{Q}^{\prime \prime}$-finite if $\mathcal{P}(X)$ is $\mathcal{Q}$-finite.

REMARKS 2. 1. Spišiak and Vojtáš [SV88] notice that IV" is equivalent to III, and also that III $^{\prime \prime}$ is equivalent to I (by a result of Tarski [Tar24] which states that for each infinite set $X, \mathcal{P}(\mathcal{P}(X))$ is IV-infinite). As a consequence, $\mathrm{I}^{\prime \prime}, \mathrm{Ia}^{\prime \prime}$, and $\mathrm{II}^{\prime \prime}$ are also equivalent to $\mathrm{I}$.

2. As noted by Spišiak [Spi93], in any model of ZFA where $\mathcal{P}(\omega)$ is not well orderable, all sets are VII"-finite (while every infinite ordinal is VII-infinite). Of course, this makes $\mathrm{VII}^{\prime \prime}$ a rather unreasonable definition for finiteness, but it still satisfies the formal requirement of being equivalent to notion I under AC. This illustrates the fact that not all the notions mentioned in this paper can be considered reasonable definitions of finiteness.

Another group of notions of finiteness was introduced by Truss [Tru74] in the form of classes of Dedekind finite cardinals.

Definition 3. Define the following classes of cardinals:

$\Delta_{1}=\{\mathfrak{a}: \mathfrak{a}=\mathfrak{b}+\mathfrak{c} \rightarrow \mathfrak{b}$ or $\mathfrak{c}$ is finite $\}$,

$\Delta_{2}=\{|X|$ : any linearly ordered partition of $X$ is finite $\}$,

$\Delta_{3}=\{|X|$ : any linearly ordered subset of $X$ is finite $\}$,

$\Delta_{4}=\left\{\mathfrak{a}: \neg\left(\aleph_{0} \leq^{*} \mathfrak{a}\right)\right\}$

$\left(|x| \leq^{*}|y|\right.$ if there exists a function $f$ from $y$ onto $\left.x\right)$,

$\Delta_{5}=\left\{\mathfrak{a}: \neg\left(\mathfrak{a}+1 \leq^{*} \mathfrak{a}\right)\right\}$. 
Remarks 3. 1 . We will use the symbols $\Delta_{i}$, for $i=1, \ldots, 5$, as notions of finiteness; that is, we will say that a set $X$ is $\Delta_{i}$-finite if $|X| \in \Delta_{i}$.

2. Clearly, $\Delta_{1}$-finiteness is equivalent to Ia-finiteness. It is also noticed by Truss [Tru74] that $\Delta_{2}$-finiteness is equivalent to II-finiteness and that $\Delta_{4}$-finiteness is equivalent to III-finiteness.

3. It can be shown that a set is $\Delta_{5}$-infinite if and only if it admits the structure of a tree of infinite height.

Howard and Yorke [HY89] introduced yet another notion:

Definition 4. A set $X$ is $D$-finite if either it has at most one element, or $X=X_{1} \cup X_{2}$ with $\left|X_{1}\right|,\left|X_{2}\right|<|X|$.

A $D$-finite set with more than one element is called decomposable. In the same paper, besides determining the relation between $D$ and each of the notions from Definition 1, the authors introduce the following principles: If $\mathcal{Q}_{1}, \mathcal{Q}_{2}$ are notions of finiteness, $E\left(\mathcal{Q}_{1}, \mathcal{Q}_{2}\right)$ stands for the formula $\forall X$ ( $X$ is $\mathcal{Q}_{1}$-finite $\leftrightarrow X$ is $\mathcal{Q}_{2}$-finite). Several of these principles are (or are equivalent to) well known weak principles of choice (see Howard and Rubin [HR98], Note 94). In particular, $E(\mathrm{~V}, \mathrm{VI}), E(\mathrm{VI}, \mathrm{VII})$, and $E(\mathrm{I}, D)$ are equivalent to AC.

It should be remarked that several other notions of finiteness have been studied by other researchers (for example, see Tarski [Tar38]); however, the ones listed here seem to be among the most commonly studied, and we will concentrate our work on them.

2.1. Implications between notions of finiteness. Given two notions of finiteness $\mathcal{Q}_{0}$ and $\mathcal{Q}_{1}$, we use the abbreviation $\mathcal{Q}_{0} \rightarrow \mathcal{Q}_{1}$ instead of the formula $\forall X$ ( $X$ is $\mathcal{Q}_{0}$-finite $\rightarrow X$ is $\mathcal{Q}_{1}$-finite).

It is shown in Lévy [Lév58], building on results of Tarski [Tar24], that

$$
\mathrm{I} \rightarrow \mathrm{Ia} \rightarrow \mathrm{II} \rightarrow \mathrm{III} \rightarrow \mathrm{IV} \rightarrow \mathrm{V} \rightarrow \mathrm{VI} \rightarrow \mathrm{VII} .
$$

It is easy to see that if $\mathcal{Q}_{0}, \mathcal{Q}_{1}$ are notions of finiteness and $\mathcal{Q}_{0} \rightarrow \mathcal{Q}_{1}$, then $\mathcal{Q}_{0}^{\prime \prime} \rightarrow \mathcal{Q}_{1}^{\prime \prime}$. Also, we have VII $\rightarrow \mathrm{VII}^{\prime \prime}:$ If $X$ is not well orderable, then $\mathcal{P}(X)$ is not well orderable, since $X$ can be mapped one-to-one into $\mathcal{P}(X)$. Besides this, Spišiak and Vojtáš [SV88] show, using elementary cardinal arithmetic, that $\mathrm{III} \rightarrow \mathrm{V}^{\prime \prime}, \mathrm{V}^{\prime \prime} \rightarrow \mathrm{IV}$, and $\mathrm{VI}^{\prime \prime} \rightarrow \mathrm{V}$. These derived notions are further studied by Spišiak [Spi93] and Howard and Spišiak [HS].

Truss [Tru74] proves that the following implications are theorems of ZFA: $\mathrm{III} \rightarrow \Delta_{5} \rightarrow$ IV and II $\rightarrow \Delta_{3} \rightarrow$ IV

Howard and Yorke prove that IV $\rightarrow D \rightarrow$ VII.

Finally, our last implication is stated in the following theorem, which has a surprisingly simple proof:

Theorem 5 . V" $\rightarrow \Delta_{5}$. 
Proof. Suppose that $X$ is not $\Delta_{5}$-finite. Then there exists a function $f$ from $X$ onto $X \cup\{u\}$, where $u \notin X$. Now we have a one-to-one map $Y \mapsto f^{-1}(Y)$ from $\mathcal{P}(X \cup\{u\})$ into $\mathcal{P}(X)$; this means that

$$
2^{|X|} \geq 2^{|X|+1}=2 \cdot 2^{|X|} .
$$

Therefore $\mathcal{P}(X)$ is $\mathrm{V}$-infinite and $X$ is $\mathrm{V}^{\prime \prime}$-infinite.

These implications are summarized in Figure 1.

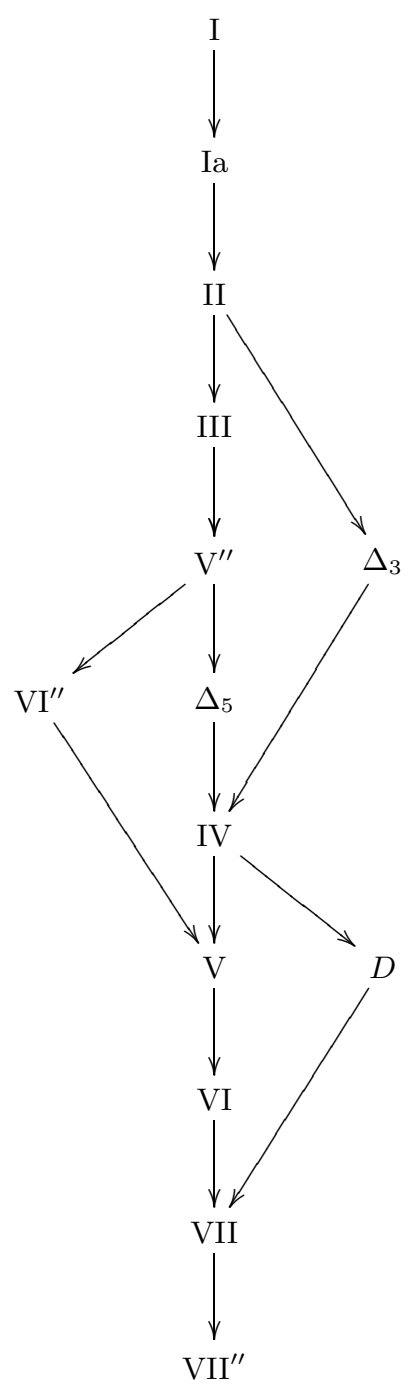

Fig. 1. Relations between notions of finiteness 
2.2. Independence results about notions of finiteness. Here we show that the implications in Figure 1 (and those that can be derived by transitivity) are the only ones that hold in general.

Independence results in this article will be obtained using two well known methods: permutation models (for ZFA) and symmetric models (for ZF). For lack of space we will not describe the construction of most of the models used, especially when the models have been thoroughly described in the literature.

In what follows, $A$ will always be the set of atoms of the universe under discussion, except in Section 4.2, where it will be a set of reals.

Lévy [Lév58] proved that Ia $\nrightarrow \mathrm{I}$ (using the set $A$ in the Basic Fraenkel Model), that II $\nrightarrow$ Ia (considering the set $2 \times A$ in the same model), and that $\mathrm{VI} \nrightarrow \mathrm{V}$ (using the set $\omega \times A$ in Mostowski's ordered model; this way he proves the independence of the notions even under the assumption of the Linear Ordering Principle).

Truss [Tru74] proved that III $\nrightarrow \Delta_{3}$ (using the set $A$ in Mostowski's ordered model); as a consequence we find that III $\nrightarrow$ II, and that $\mathcal{Q} \nrightarrow \Delta_{3}$ for any notion $\mathcal{Q}$ below III in Figure 1. Also, it is proved there that $\Delta_{3} \nrightarrow \Delta_{5}$, using the Second Fraenkel Model. Consequently, $\Delta_{3} \nrightarrow \mathcal{Q}$ for any notion $\mathcal{Q}$ above $\Delta_{5}$.

Howard and Yorke [HY89] proved that $D \nrightarrow$ VI, using the set of all finite, one-to-one sequences from $A \cup \omega$ in the Basic Fraenkel Model. That means that $D \nrightarrow \mathcal{Q}$ is false unless $\mathcal{Q}$ is VII or $\mathrm{VII}^{\prime \prime}$.

As we mentioned before, Spišiak [Spi93] showed that VII" $\nrightarrow$ VII (see Remark 2.2), and therefore $\mathrm{VII}^{\prime \prime} \nrightarrow \mathcal{Q}$ for all other $\mathcal{Q}$ in Figure 1. Also, Howard and Spišiak $[\mathrm{HS}]$ prove that $\mathrm{V}^{\prime \prime} \nrightarrow$ III, which implies $\mathcal{Q}_{0} \nrightarrow \mathcal{Q}_{1}$ for every $\mathcal{Q}_{0}$ below $\mathrm{V}^{\prime \prime}$ and every $\mathcal{Q}_{1}$ above III. The model used is rather complicated, and we will not offer a description for lack of space.

The next two lemmas will be enough to eliminate the rest of the implications that do not appear in Figure 1.

LEMMA $6 . \Delta_{5} \nrightarrow \mathrm{VI}^{\prime \prime}$ and $\Delta_{3} \nrightarrow \mathrm{VI}^{\prime \prime}$.

Proof. Consider the set $A$ in the Second Fraenkel Model. Truss [Tru74] proves that $A$ is $\Delta_{5}$-finite, while Howard and Spišiak [HS] prove that $A$ is $\mathrm{VI}^{\prime \prime}$-infinite by explicitly defining a one-to-one function $f: \mathcal{P}(A) \times \mathcal{P}(A) \rightarrow$ $\mathcal{P}(A)$ in the model.

To prove the second part, it is enough to prove that $A$ is also $\Delta_{3}$-finite. It is easy to see that a linear order for an infinite subset of $A$ cannot have a finite support.

Consequently, we have $\mathcal{Q} \nrightarrow \mathrm{VI}^{\prime \prime}$ for every $\mathcal{Q}$ below $\Delta_{5}$ (in particular, $\left.D \nrightarrow \mathrm{VI}^{\prime \prime}\right)$. 
LEMMA $7 . \mathrm{VI}^{\prime \prime} \nrightarrow D$.

Proof. We use model M6 from Howard and Yorke [HY89]. The model is constructed as follows: Start with a model of ZFA + AC and consider the set $A$ of atoms to be ordered in the order type of the rationals. M6 is the permutation model obtained using the group of all permutations that move at most finitely many elements of $A$, and the ideal of bounded subsets of $A$ as the normal ideal of supports.

Howard and Yorke [HY89] prove that $A$ is not $D$-finite in $M 6$. We will prove here that $A$ is $\mathrm{VI}^{\prime \prime}$-finite.

For the sake of a contradiction, suppose that there is a one-to-one map $f$ from $\mathcal{P}(A) \times \mathcal{P}(A)$ into $\mathcal{P}(A)$; let $E$ be a support for $f$. Choose distinct $a, b \in A \backslash E$, and let $X=f(\{a\},\{b\})$. Then we can assume, without loss of generality, that $\{a\} \neq X \backslash E$ and $\{a\} \neq A \backslash(X \cup E)$ (if this fails for $a$, then it is true for $b$ ). Then we can find $c \in A \backslash E, c \neq a$, such that the transposition $(a, c)$ leaves $X$ invariant. In that case we conclude that $f(c, b)=X$, and therefore $f$ is not one-to-one.

As a consequence of this lemma, we obtain $\mathrm{VI}^{\prime \prime} \nrightarrow \mathcal{Q}$ for each $\mathcal{Q}$ above $D$; in particular, we have $\mathrm{VI}^{\prime \prime} \nrightarrow \mathrm{IV}$.

2.3. Two additional lemmas. Before we finish this section we establish the following lemmas.

Lemma 8. If $X$ is $\mathcal{Q}$-finite, where $\mathcal{Q}$ is any of the notions in Figure 1 , and $|X|=\left|X^{\prime}\right|$, then $X^{\prime}$ is $\mathcal{Q}$-finite.

Lemma 8 , which can be easily verified case by case, states that all our notions of finiteness are cardinal invariants, as should be expected.

Lemma 9. For every notion $\mathcal{Q}$ of finiteness in Figure 1,

$$
X \text { is } \mathcal{Q} \text {-finite if and only if } X \cup\{y\} \text { is } \mathcal{Q} \text {-finite, }
$$

where $X$ is any set and $y$ is any object (set or atom).

Proof. For notions $\mathcal{Q}$ such that IV $\rightarrow \mathcal{Q}$, we find that if either $X$ or $X \cup\{y\}$ is $\mathcal{Q}$-infinite, then $|X|=|X \cup\{y\}|$, and therefore the contrapositive of (2) holds.

For the notions that are not implied by IV, (2) can be easily verified case by case. We show here the proof for $\mathcal{Q}=\mathrm{V}^{\prime \prime}$; the case $\mathcal{Q}=\mathrm{VI}^{\prime \prime}$ is similar. We assume that $y \notin X$ to avoid trivialities.

Since $2^{|X|+1}=2 \cdot 2^{|X|}$, we see that $\mathcal{P}(X)$ is $\mathrm{V}$-infinite if and only if $\mathcal{P}(X \cup\{y\})$ is V-infinite; one of the directions uses the fact that if $2 \mathfrak{a}=2 \mathfrak{b}$ then $\mathfrak{a}=\mathfrak{b}$ for all infinite cardinals $\mathfrak{a}, \mathfrak{b}$; this theorem can be proved without $\mathrm{AC}$ (see Jech [Jec73, Thm. 11.9]). Consequently, $X$ is $\mathrm{V}^{\prime \prime}$-infinite if and only if $X \cup\{y\}$ is $\mathrm{V}^{\prime \prime}$-infinite. 
It should be remarked, though, that many other reasonable properties (like closure under unions, products, etc.) fail for at least some of the notions of finiteness considered in this paper.

3. Axioms of choice for finite families. In this section we define two kinds of principles of choice, stated as axioms of choice for families that are finite according to one of the notions introduced in the previous section.

3.1. Definitions and basic properties. Given a family $X$ of non-empty sets (that is, neither $\emptyset$ nor any atom is a member of $X$ ), a choice function is a function $f: X \rightarrow \bigcup X$ such that

$$
\forall x \in X(f(x) \in x) .
$$

For an indexed family $\left\{x_{j}: j \in J\right\}$ of non-empty sets, we will also call choice function a function $f$ with domain $J$ such that for all $j \in J, f(j) \in x_{j}$, as long as $f(j)=f\left(j^{\prime}\right)$ whenever $x_{j}=x_{j^{\prime}}$.

A partial choice function for an infinite family $X$ is a choice function for a infinite subfamily of $X$.

Definition 10. Let $\mathcal{Q}$ be a notion of finiteness. The principle $C(\mathcal{Q})$ is the sentence:

$\forall X$ ( $X$ is a $\mathcal{Q}$-finite family of non-empty sets $\rightarrow X$ has a choice function). The principle $C^{-}(\mathcal{Q})$ is the sentence:

$\forall X(X$ is a $\mathcal{Q}$-finite but infinite family of non-empty sets

$$
\rightarrow X \text { has a partial choice function). }
$$

REMARKs 4. The following are all theorems of ZFA:

1. $C(\mathrm{I})$ is true (this is just a restatement of (1) from the Introduction); it can be proved by an induction argument. Also, $C^{-}(\mathrm{I})$ is vacuously true.

2. If $\mathcal{Q}$ is a notion of finiteness, then $C(\mathcal{Q})$ implies $C^{-}(\mathcal{Q})$.

3. If $\mathcal{Q}_{1}, \mathcal{Q}_{2}$ are notions of finiteness and $\mathcal{Q}_{1} \rightarrow \mathcal{Q}_{2}$, then $C\left(\mathcal{Q}_{2}\right)$ implies $C\left(\mathcal{Q}_{1}\right)$ and $C^{-}\left(\mathcal{Q}_{2}\right)$ implies $C^{-}\left(\mathcal{Q}_{1}\right)$.

4. The principle $E(\mathrm{I}, \mathcal{Q})$ implies $C(\mathcal{Q})$ for every notion of finiteness $\mathcal{Q}$.

Lemma 11. If $C(\mathcal{Q})$ holds and $\mathcal{P}(X)$ is $\mathcal{Q}$-finite, then $X$ is well orderable.

Proof. By Lemma 9, $\mathcal{P}(X) \backslash\{\emptyset\}$ is $\mathcal{Q}$-finite. Once we have a choice function for $\mathcal{P}(X) \backslash\{\emptyset\}$, it is enough to repeat the usual proof of the fact that AC implies the Well Ordering Principle.

Corollary 12. $C(\mathrm{VII})$ and $C\left(\mathrm{VII}^{\prime \prime}\right)$ are equivalent to $\mathrm{AC}$.

Proof. Clearly, $\mathrm{AC}$ implies $C\left(\mathrm{VII}^{\prime \prime}\right)$ and $C\left(\mathrm{VII}^{\prime \prime}\right)$ implies $C(\mathrm{VII})$. Now assume $C$ (VII) and suppose $X$ is a non-well-orderable set; then $\mathcal{P}(X)$ is also 
non-well-orderable, and by Lemma 11 we conclude that $X$ is actually well orderable, a contradiction.

COROLlary 13. If $\mathcal{Q}$ is one of the notions of finiteness in Figure 1 , and $C(\mathcal{Q})$ holds, then we have $E\left(\mathrm{I}, \mathcal{Q}^{\prime \prime}\right)$.

Proof. Suppose $C(\mathcal{Q})$ holds and that $X$ is $\mathcal{Q}^{\prime \prime}$-finite but infinite. Since $\mathcal{P}(X)$ is $\mathcal{Q}$-finite, Lemma 11 guarantees that $X$ is well orderable.

Being infinite and well orderable, $X$ cannot be VII-finite, and therefore, it is not finite according to any of the other definitions in Figure 1, except VII". Therefore, the only case when this can happen is when $\mathcal{Q}$ is VII. However, the assumption $C(\mathrm{VII})$ is equivalent to $\mathrm{AC}$, and this implies $E\left(\mathrm{I}, \mathrm{VII}^{\prime \prime}\right)$.

3.2. Additional implications. We begin with an easy result:

LEMMA 14. $C(\mathrm{Ia})$ and $C^{-}(\mathrm{Ia})$ are equivalent.

Proof. We know that $C(\mathrm{Ia})$ implies $C^{-}(\mathrm{Ia})$. To prove the converse implication, assume that $X$ is an infinite Ia-finite family of non-empty sets and let $f$ be a partial choice function for $X$ with (infinite) domain $X^{\prime} \subset X$. Since $X \backslash X^{\prime}$ is finite, it has a choice function $g$. Then $f \cup g$ is a choice function for $X$.

The preceding result can be proved in ZFA. However, it is vacuously true in $\mathrm{ZF}$, as the next two results show. We will see in Section 4 that it is not vacuously true in ZFA, by establishing that Theorem 15 and Corollary 16 cannot be proved in ZFA.

THEOREM $15(\mathrm{ZF})$. Let $X$ be an infinite set. If every family of nonempty sets indexed by $X$ has a partial choice function, then $X$ is partible (that is, Ia-infinite).

Proof. For the sake of a contradiction, suppose that $X$ is Ia-finite. Since every subset of $X$ is either finite or the complement of a finite set, when a formula holds for infinitely many elements of $X$, we will say that the formula is true "for almost all $x \in X$ " or "almost everywhere".

Given any function $f$ defined almost everywhere on $X$, we define $\varrho(f)$ as the unique ordinal $\alpha$ such that $\operatorname{rank}(f(x))=\alpha$ for almost all $x \in X$ (such $\alpha$ exists because any well ordered partition of $X$ has exactly one infinite part). If $f, g$ are functions defined almost everywhere on $X$, we will write $g \prec f$ if $g(x) \in f(x)$ for almost all $x \in X$. Clearly, if $g \prec f$, then $\varrho(g)<\varrho(f)$.

Choose a function $f_{0}$ with minimum $\varrho$ such that range $\left(f_{0}\right)$ is infinite (there is at least one such function with infinite range, namely, the identity function on $X)$; we can assume that $\emptyset \notin \operatorname{range}\left(f_{0}\right)$.

Let $K=\left\{c: c \in f_{0}(x)\right.$ for almost all $\left.x \in X\right\}$, and let $f_{1}(x)=f_{0}(x) \triangle K$ for all $x \in \operatorname{dom}\left(f_{0}\right)$ ( $\triangle$ is the symmetric difference). If $f_{1}(x)=\emptyset$ for almost every $x \in X$, then $f_{0}(x)=K$ for almost all $x \in X$, contradicting the fact 
that range $\left(f_{0}\right)$ is infinite. Therefore, $f_{1}$ is a family of non-empty sets defined almost everywhere on $X$.

Since we can see $f_{1}$ as a family of sets indexed by $X$ (by taking any undefined values of $f_{1}$ to be an arbitrary non-empty set), by hypothesis there exists a partial choice function $g_{1}$ defined almost everywhere on $X$; that is, $g_{1} \prec f_{1}$.

Now, for all $c \in K$ we have $\operatorname{rank}(c)<\varrho\left(f_{0}\right)$. Therefore $\varrho\left(g_{1}\right)<\varrho\left(f_{0}\right)$, since the images of $g_{1}$ are either in $K$ or in $f_{0}(x)$ for some $x \in X$. Thus, $g_{1}$ has finite range, and consequently it is constant almost everywhere with value, say, $c_{1}$.

We arrive at a contradiction as follows: $c_{1} \in K$ if and only if $c_{1} \in f_{0}(x)$ for almost all $x \in X$. Therefore, $c_{1} \notin f_{0}(x) \triangle K=f_{1}(x)$ for almost all $x \in X$. This is not possible, since $g_{1} \prec f_{1}$.

Corollary $16(\mathrm{ZF}) . C^{-}(\mathrm{Ia})$ is equivalent to $E(\mathrm{I}, \mathrm{Ia})$.

Proof. As we have already seen, if $E(I, I a)$ holds, then $C^{-}(\mathrm{Ia})$ is vacuously true. Suppose that $C^{-}$(Ia) holds and that $X$ is an infinite Ia-finite set. By Theorem 15, there exists a family $f: X \rightarrow V$ of non-empty sets indexed by $X$ with no partial choice function.

If $f$ is constant almost everywhere with value, say, $c$, and $d \in c$, then the function $g$, constantly equal to $d$ and with domain equal to $f^{-1}(c)$, would be a partial choice function for $f$. Thus, $f$ cannot be constant almost everywhere, and therefore it must have an infinite range. But then range $(f)$ is a Ia-finite set, since any partition of range $(f)$ in two infinite sets induces a partition of $X$ in two infinite sets. The hypothesis of this corollary implies that the set range $(f)$ has a partial choice function, which means that the family $f$ has a partial choice function, a contradiction.

Unlike the previous result, the next two theorems can be proved in ZFA.

TheOREM 17. $C(\mathrm{IV})$ is equivalent to $E(\mathrm{I}, \mathrm{IV})$.

Proof. Suppose that $C(\mathrm{IV})$ holds, and that $X$ is an infinite, IV-finite set. Consider the set $X_{*}^{<\omega}$ of all finite sequences from $X$ that do not repeat elements.

It was proved by Tarski (see Lévy [Lév65, p. 225]) that $X_{*}^{<\omega}$ is IV-finite. Therefore, the family

$$
F=\left\{X_{s}=(X \backslash \operatorname{range}(s)) \times\{s\}: s \in X_{*}^{<\omega}\right\}
$$

is also IV-finite, because the map $s \mapsto X_{s}$ is one-to-one. Therefore, by hypothesis, $F$ has a choice function $f$.

We find a contradiction by defining a non-repeating $\omega$-sequence from elements of $X$, thereby showing that it cannot be IV-finite. Choose an initial element $x_{0} \in X$; the rest of the sequence is defined by recursion. Assuming 
that $\left\langle x_{0}, \ldots, x_{k}\right\rangle$ has already been defined, we define $x_{k+1}$ by

$$
x_{k+1}=\left(f\left(X_{\left\langle x_{0}, \ldots, x_{k}\right\rangle}\right)\right)_{1} .
$$

The same result can be obtained for $\Delta_{3}$, using the same proof. In this case, the fact that $X_{*}^{<\omega}$ is $\Delta_{3}$-finite whenever $X$ is $\Delta_{3}$-finite is due to Truss [Tru74].

TheOrem 18. $C\left(\Delta_{3}\right)$ is equivalent to $E\left(\mathrm{I}, \Delta_{3}\right)$.

The implications found in this section are summarized in Figure 2.

\section{Independence results for axioms of choice for finite fami-} lies. Our independence results come from three sources: the Basic Fraenkel Model, the Basic Cohen Model, and the Jech-Sochor embedding models for several permutation models.

4.1. The Basic Fraenkel Model (BFM). It is established by Spišiak and Vojtáš [SV88] and Spišiak [Spi93] that for most pairs $\mathcal{Q}, \mathcal{Q}^{\prime}$ of different notions of finiteness from Figure 1, there is a set in BFM that is finite according to one of the two notions, but not according to the other. Two exceptions are the pairs VII, VII" (see Corollary 20) and II, III (see Corollary 22.3); this second exception answers a question of Spišiak [Spi93]. Below we will establish two results about principles from Section 3 which hold in BFM. First, we need some lemmas. The first one contains well known properties of permutation models defined using supports:

LEMMA 19. Let $\mathcal{N}$ be a permutation model defined using supports. Then:

1. If $E$ supports $x$, and $\pi, \pi^{\prime}$ are permutations of $A$ such that $\pi\left\lceil E=\pi^{\prime} \uparrow E\right.$, then $\pi x=\pi^{\prime} x$.

2. For all $x \in \mathcal{N}, x$ is well orderable in $\mathcal{N}$ if and only if there exists $E \in I$ that supports every element of $x$.

3. For every set $x \in \mathcal{N}$, if $x$ is well orderable, then $\mathcal{P}(x)$ is well orderable.

Corollary 20. BFM satisfies E(VII, VII").

LEMMA 21. The model BFM has the following property: Suppose that $E \cup\{a\}$ is a support for some object $x$, and suppose that there is a permutation $\pi$ of $A$ such that $\pi \in \operatorname{fix}(E), \pi x=x$, and $\pi a \neq a$. Then $E$ is a support for $x$.

Proof. Let $\sigma \in \operatorname{fix}(E)$. If $\sigma a=a$, then $\sigma x=x$; assume then that $\sigma a \neq a$. Therefore, since $\pi a, \sigma a \notin E \cup\{a\}$, the transposition $(\pi a, \sigma a)$ is in fix $(E \cup\{a\})$; also, $\sigma\lceil E \cup\{a\}=(\pi a, \sigma a) \pi\lceil E \cup\{a\}$, so, by Lemma 19.1, 


$$
\sigma x=(\pi a, \sigma a) \pi x=(\pi a, \sigma a) x=x .
$$

Therefore, $\operatorname{fix}(E) \subset(x)$.

COROLlary 22. The following hold in BFM:

1. (Blass [Bla77]) Every non-well-orderable set contains a one-to-one image of an infinite subset of $A$.

2. If a set can be linearly ordered, then it can be well ordered.

3. $E(\mathrm{II}, \mathrm{III})$ and $E\left(\Delta_{3}, \mathrm{IV}\right)$.

Proof. For clause 1, let $X$ be a non-well-orderable set in BFM, $E$ a support for $X$, and $x_{0}$ an element of $X$ not supported by $E$ (see Lemma 19.2). Take $E^{\prime} \cup\left\{a_{0}\right\}$ to be a support for $x_{0}$ of minimum size containing $E$, with $a_{0} \notin E$. Then $\left\{\left\langle\pi a_{0}, \pi x_{0}\right\rangle: \pi \in \operatorname{fix}\left(E^{\prime}\right)\right\}$ is a function with domain $A \backslash E^{\prime}$, and Lemma 21 guarantees that it is one-to-one. Clause 2 is obtained directly from 1. Clause 3 follows from 2: if a set has no infinite well orderable partitions, then it has no infinite linearly orderable partitions; and a set contains an infinite linearly orderable subset if and only if it contains an infinite well orderable subset.

TheOREM 23. $C^{-}\left(\mathrm{VII}^{\prime \prime}\right)$ holds in BFM.

Proof. By Corollary 20, it is enough to prove $C^{-}$(VII).

Let $X$ be a non-well-orderable (that is, VII-finite but infinite) family of non-empty sets; we want to prove that there exists an infinite subset $X^{\prime} \subset X$ with a choice function.

Let $E$ be a support for $X$. By Lemma 19.2, there exists $x_{0} \in X$ that is not supported by $E$; let $E_{0}$ be a support for $x_{0}$ of minimum size such that $E \subset E_{0}$.

Since $x_{0} \neq \emptyset$, we can choose an element $y_{0} \in x_{0}$; let $E_{1}$ be a support for $y_{0}$ such that $E_{0} \subset E_{1}$. Pick $a_{0} \in E_{0} \backslash E$, and set $E_{2}=E_{1} \backslash\left\{a_{0}\right\}$. Define

$$
f=\left\{\left\langle\pi x_{0}, \pi y_{0}\right\rangle: \pi \in \operatorname{fix}\left(E_{2}\right)\right\} \text {. }
$$

ClaIM. $f$ is a function.

Indeed, if $\pi x_{0}=\pi^{\prime} x_{0}$ but $\pi y_{0} \neq \pi^{\prime} y_{0}$, that means that $\pi a_{0} \neq \pi^{\prime} a_{0}$, and then $\pi^{-1} \circ \pi^{\prime} x_{0}=x_{0}$, but $\pi^{-1} \circ \pi^{\prime} a_{0} \neq a_{0}$. Since $\pi^{-1} \circ \pi^{\prime} \in \operatorname{fix}\left(E_{0} \backslash\left\{a_{0}\right\}\right)$, we can apply Lemma 21 to conclude that $E_{0} \backslash\left\{a_{0}\right\}$ supports $x_{0}$; this contradicts the choice of $E_{0}$ as a support for $x_{0}$ of minimum size.

Claim. $\operatorname{dom}(f) \subset X$, and it is infinite.

In fact, for all $\pi \in \operatorname{fix}\left(E_{2}\right)$, we have $\pi x_{0} \in \pi X=X$. Also, for every $b, b^{\prime} \notin E_{2}, b \neq b^{\prime}$, the elements $\left(a_{0}, b\right) x_{0}$ and $\left(a_{0}, b^{\prime}\right) x_{0}$ must be different: Suppose they are not, and take $\pi=\left(a_{0}, b^{\prime}\right)\left(a_{0}, b\right)$. We have $\pi x_{0}=x_{0}$, while $\pi a_{0}=b \neq a$; then, using Lemma 21 , we can conclude that $E_{0} \backslash\left\{a_{0}\right\}$ supports $x_{0}$, and again this is a contradiction. 
Finally, we have

Claim. $f$ is a choice function for its domain.

Clearly, for all $\pi \in \operatorname{fix}\left(E_{2}\right), \pi y_{0} \in \pi x_{0}$.

This result shows that both Corollary 16 and Theorem 15 cannot be proved in ZFA.

Another consequence of Theorem 23 is that $C^{-}$(VII) does not imply $C$ (II) in ZFA, as can be seen from the following lemma:

LEMma 24. $C$ (II) does not hold in BFM.

Proof. We claim that the set $[A]^{2}$ of all 2-element subsets of $A$ is II-finite. Suppose it is not; then by Remark 3.2 there exists an infinite linearly ordered partition of $[A]^{2}$. Let $E$ be a support for the partition and its linear order, and take $a_{1}, a_{2}, a_{3}, a_{4} \notin E$ such that $\left\{a_{1}, a_{2}\right\}$ and $\left\{a_{3}, a_{4}\right\}$ are in different elements of the partition. Assuming that either element of the partition precedes the other leads to a contradiction, by using a permutation that interchanges the pairs $\left\{a_{1}, a_{2}\right\}$ and $\left\{a_{3}, a_{4}\right\}$ while leaving $E$ fixed.

However, $[A]^{2}$ has no choice function, as can be seen by an easy argument showing that a choice function for $[A]^{2}$ cannot be supported by a finite set.

Corollary 25. For all notions $\mathcal{Q}_{1}, \mathcal{Q}_{2}$ of finiteness in Figure 1 different from I and Ia, $C^{-}\left(\mathcal{Q}_{1}\right)$ does not imply $C\left(\mathcal{Q}_{2}\right)$ in ZFA.

4.2. The Basic Cohen Model. The model BCM has been well studied, and there are many consequences of $\mathrm{AC}$ that have been proved to hold in it. Two of them are listed in the next theorem. Notice that in this section $A$ will stand for a set of reals, and that there are no atoms in BCM.

Theorem 26. The following are true in the Basic Cohen Model $\mathcal{N}$ :

1. (Cohen) There exists a set $A \in \mathcal{N}$ of reals which is Dedekind finite.

2. (Halpern and Lévy) The Boolean Prime Ideal Theorem holds in $B C M$.

It is a consequence of Theorem 26.2 that every set can be linearly ordered in BCM. A proof of that weaker result can be found in Jech [Jec73, Section 5.5]); we will borrow the notation and some of the lemmas used there for the proof of our main result about BCM (the reader can find in that book the details we skip here).

$\mathrm{BCM}$ is constructed from a standard model $\mathcal{M}$ of ZFC by adding independent Cohen reals $x_{n}, n \in \omega$ (using the forcing notion $\mathbb{P}=\{p: p$ is a finite partial function from $\omega \times \omega$ to 2$\}$ ), and then passing to a symmetric submodel $\mathcal{N}$ of the generic extension $\mathcal{M}[G]$ using a group of automorphisms of $\mathbb{P}$ obtained from the group of all permutations of $\omega$, together with the normal filter obtained by using finite subsets of $\omega$ as supports. The enumeration 
$n \mapsto x_{n}$ is not in $\mathcal{N}$, which makes it possible for the set $A=\left\{x_{n}: n \in \omega\right\}$ to be Dedekind finite.

Like in Jech [Jec73, Section 5.5], we will assume that there is a well order of $\mathcal{M}$ in $\mathcal{M}$; that is, that there is a bijection between $\mathcal{M}$ and the class of ordinals in $\mathcal{M}$ which is a definable class in $\mathcal{M}$. This assumption is not really necessary, since all the uses of this well order of the universe can be relativized to some set large enough to contain all the objects studied; however, this form of global choice allows the arguments to run more smoothly.

We say that $e \subset \omega$ is a support for a name $\dot{x}$ if fix $(e) \subset(\dot{x})$. It can be checked that the intersection of two supports for a name is also a support for that name; therefore, for each name $\dot{x}$ there exists a least support $s(\dot{x})$. Notice that if $\pi\left\lceil s(\dot{x})=\varrho \mid s(\dot{x})\right.$, then $\pi(\dot{x})=\varrho(\dot{x})$, since $\varrho^{-1} \pi \in$ fix $(s(\dot{x}))$.

For each finite subset $E \subset A$ we have a canonical name $\dot{E}: \dot{E}\left(\dot{x}_{n}\right)=1$ for all $x_{n} \in E$. We say that $\dot{E}$ is a support for $\dot{x}$ if $E=\left\{x_{n_{1}}, \ldots, x_{n_{k}}\right\}$ and $\left\{n_{1}, \ldots, n_{k}\right\}$ supports $\dot{x}$. It can be checked that the class $\dot{\Delta}=\left\{(\dot{E}, \dot{x})^{\cdot}\right.$ : $\dot{E}$ supports $\dot{x}\}$ is a symmetric class, and therefore its interpretation is a class $\Delta$ in $\mathcal{N}$. This way we can say, in $\mathcal{N}$, that $E$ is a support of $x$ if $\Delta(E, x)$.

Lemma 27. Every $x \in \mathcal{N}$ has a minimum support.

Lemma 28. In $\mathcal{N}$, there is a one-to-one function $\mathcal{F}: \mathcal{N} \rightarrow[A]^{<\omega} \times$ Ord.

Sketch of proof. We take $\mathcal{F}=\left(\mathcal{F}_{1}, \mathcal{F}_{2}\right)$, where $\mathcal{F}_{1}$ assigns to $x$ its minimum support and $\mathcal{F}_{2}$ assigns to $x$ the minimum ordinal corresponding to a name $\dot{y}$ such that $i_{G}(\pi \dot{y})=x$ for some permutation $\pi$ of $\omega$ (in a fixed enumeration in $\mathcal{M}$ of the class of names, constructed using the well order of $\mathcal{M})$.

Lemma 29. For every formula $\varphi$ and sets $y_{1}, \ldots, y_{k}$, there exists $m \in \omega$ such that, if

$$
E=\bigcup_{j=1}^{k} \mathcal{F}_{1}\left(y_{j}\right)=\left\{x_{1}, \ldots, x_{n}\right\},
$$

then for every permutation $\pi$ of $A$ which satisfies

$$
\pi x_{i} \cap m=x_{i} \cap m, \quad i=1, \ldots, n,
$$

there exist $y_{1}^{\prime}, \ldots, y_{k}^{\prime}$ such that:

1. $\mathcal{F}\left(y_{j}^{\prime}\right)=\left(\pi \cdot \mathcal{F}_{1}\left(y_{j}\right), \mathcal{F}_{2}\left(y_{j}\right)\right)$ for $j=1, \ldots, k$.

2. $\mathcal{N} \vDash \varphi\left[y_{1}, \ldots, y_{k}\right] \leftrightarrow \varphi\left[y_{1}^{\prime}, \ldots, y_{k}^{\prime}\right]$.

Proof. Without loss of generality we can assume that $\mathcal{N} \vDash \varphi\left[y_{1}, \ldots, y_{k}\right]$ (otherwise we can consider the formula $\neg \varphi$ ). Then there exist $p \in G$ and names $\dot{y}_{1}, \ldots, \dot{y}_{k}$ (which we choose so that they have the smallest possible supports) such that $i_{G}\left(\dot{y}_{j}\right)=y_{j}$ for $j=1, \ldots, k$, and

$$
p \Vdash \varphi^{\mathcal{N}}\left[\dot{y}_{1}, \ldots, \dot{y}_{k}\right] .
$$


Let $e=s\left(\dot{y}_{1}\right) \cup \ldots \cup s\left(\dot{y}_{k}\right)$. By Lemma 5.24 in Jech [Jec73], we can assume that $\operatorname{dom}(p) \subset e \times \omega$. Let $m \in \omega$ be the least such that $\operatorname{dom}(p) \subset e \times m$. We can assume that actually $\operatorname{dom}(p)=e \times m$.

Let $E=\left\{x_{i}: i \in e\right\}$. It is clear that $\mathcal{F}_{1}\left(y_{j}\right) \subset E$ for $j=1, \ldots, k$. Let also $\pi$ be a permutation of $A$ in $\mathcal{N}$; then there is a permutation $\sigma$ of $\omega$ in $\mathcal{M}[G]$ such that

$$
\pi x_{i}=x_{\sigma i} \quad \text { for all } i \in \omega
$$

(it is not necessary to worry about which permutations of $A$ exist in $\mathcal{N}$, since we are always interested only in moving finitely many elements).

If $\pi x_{i} \cap m=x_{i} \cap m$, for $i=1, \ldots, n$, that fact must be forced by a condition $p^{\prime} \in G$, and such a condition must extend $\sigma p$; therefore, $\sigma p \in G$. Since

$$
\sigma p \Vdash \varphi^{\mathcal{N}}\left[\sigma \dot{y}_{1}, \ldots, \sigma \dot{y}_{k}\right],
$$

taking $y_{j}^{\prime}=i_{G}\left(\sigma \dot{y}_{j}^{\prime}\right)$, for $j=1, \ldots, k$, we have $\mathcal{M}[G] \vDash \varphi^{\mathcal{N}}\left[y_{1}^{\prime}, \ldots, y_{k}^{\prime}\right]$; in other words,

$$
\mathcal{N} \vDash \varphi\left[y_{1}, \ldots, y_{k}\right] \rightarrow \varphi\left[y_{1}^{\prime}, \ldots, y_{k}^{\prime}\right] .
$$

To complete the proof of clause 2 , it is enough to repeat the argument above starting with $\mathcal{N} \vDash \varphi\left[y_{1}^{\prime}, \ldots, y_{k}^{\prime}\right]$ and using $\pi^{-1}$.

All that is left is to check that $y_{1}^{\prime}, \ldots, y_{k}^{\prime}$ satisfy the condition required in clause 1 . Indeed, for each $j=1, \ldots, k$ we see that $\sigma \dot{y}_{j}$ is in the same orbit as $\dot{y}_{j}$, therefore $\mathcal{F}_{2}\left(y_{j}^{\prime}\right)=\mathcal{F}_{2}\left(y_{j}\right)$; also, the minimum support of $\sigma \dot{y}_{j}$ is the image under $\sigma$ of the minimum support of $\dot{y}_{j}$, and consequently the minimum support of $y_{j}^{\prime}$ is the image under $\pi$ of the minimum support of $y_{j}$.

Now we can prove what will be the main property of BCM for us in this section:

TheOrem 30. $C^{-}$(VII) holds in BCM.

Proof. This proof follows ideas similar to those of the proof of Theorem 23.

Let $Y$ be a non-well-orderable set in $\mathcal{N}$. We want to show that there is an infinite subset of $Y$ with a choice function.

If for every $y \in Y$ we have $\mathcal{F}_{1}(y) \subset \mathcal{F}_{1}(Y)$, then fixing a linear order for $\mathcal{F}_{1}(Y)$ we obtain a well order for $Y$ using the function $\mathcal{F}$. Therefore, there exists $y_{0} \in Y$ such that $\mathcal{F}_{1}\left(y_{0}\right) \not \subset \mathcal{F}_{1}(Y)$. Since the empty set has empty support, there exists $z_{0} \in y_{0}$.

Consider the formula $\varphi\left(v_{1}, v_{2}, v_{3}\right)$ given by $v_{1} \in v_{2} \in v_{3}$. Then $\mathcal{N} \vDash$ $\varphi\left[z_{0}, y_{0}, Y\right]$; let $m$ be the number obtained from Lemma 29. Choose $x_{n_{0}} \in$ $\mathcal{F}_{1}\left(y_{0}\right) \backslash \mathcal{F}_{1}(Y)$, and let $E=\mathcal{F}_{1}(Y) \cup \mathcal{F}_{1}\left(y_{0}\right) \cup \mathcal{F}_{1}\left(z_{0}\right) \backslash\left\{x_{n_{0}}\right\}$. Then, for every $x \in A \backslash E$, define $\pi_{x}$ as the transposition $\left(x_{n_{0}}, x\right)$. Define

$f=\left\{\left\langle\mathcal{F}^{-1}\left(\pi_{x}\right.\right.\right.$ " $\left.\left.\left.\mathcal{F}_{1}\left(y_{0}\right), \mathcal{F}_{2}\left(y_{0}\right)\right), \mathcal{F}^{-1}\left(\pi_{x} " \mathcal{F}_{1}\left(z_{0}\right), \mathcal{F}_{2}\left(z_{0}\right)\right)\right\rangle: x \cap m=x_{n_{0}} \cap m\right\}$. 
By Lemma 29, for each such $x$,

$$
\mathcal{F}^{-1}\left(\pi_{x} " \mathcal{F}_{1}\left(z_{0}\right), \mathcal{F}_{2}\left(z_{0}\right)\right) \in \mathcal{F}^{-1}\left(\pi_{x} " \mathcal{F}_{1}\left(y_{0}\right), \mathcal{F}_{2}\left(y_{0}\right)\right) \in Y
$$

(in particular, the inverse images are defined). By a genericity argument, there exist infinitely many sets $x \in A$ such that $x \cap m=x_{n_{0}} \cap m$. Since $\mathcal{F}$ is one-to-one, if $x \neq x^{\prime}$ and $x^{\prime} \cap m=x \cap m=x_{n_{0}} \cap m$, then

$$
\mathcal{F}^{-1}\left(\pi_{x} \text { ' } \mathcal{F}_{1}\left(y_{0}\right), \mathcal{F}_{2}\left(y_{0}\right)\right) \neq \mathcal{F}^{-1}\left(\pi_{x^{\prime}} \text { ' } \mathcal{F}_{1}\left(y_{0}\right), \mathcal{F}_{2}\left(y_{0}\right)\right) .
$$

Therefore, $f$ is a choice function with an infinite domain contained in $Y$.

Theorem 31. In $\mathrm{ZF}$ it cannot be proved that $C^{-}(\mathrm{VII}), C\left(\Delta_{3}\right)$, and $C(\mathrm{III})$, even jointly, imply $C\left(\Delta_{5}\right)$ or $C^{-}\left(\mathrm{VII}^{\prime \prime}\right)$.

Proof. We use BCM. By Theorem 30, $C^{-}$(VII) holds in BCM; it is also known that $E(\mathrm{I}, \mathrm{III})$ holds there (see Truss [Tru74]), as well as $E\left(\mathrm{I}, \Delta_{3}\right)$, since every set can be linearly ordered in BCM. Therefore $C\left(\Delta_{3}\right)$ and $C$ (III) hold in BCM.

However, it is proved in Truss [Tru74] that $A \in \mathcal{N}$ is a $\Delta_{5}$-finite set. Therefore the set $\{A \cap(-\infty, x): x \in A\}$ is also $\Delta_{5}$, but it has no choice function (otherwise we can find a countable subset of $A$ ). This means that $C\left(\Delta_{5}\right)$ fails in BCM. Also, $\omega$ is VII"-finite in BCM; since partial choice for countable families is equivalent to countable choice, and countable choice fails in BCM, $C^{-}\left(\mathrm{VII}^{\prime \prime}\right)$ fails in BCM.

It is not clear at the moment whether $C\left(\mathrm{~V}^{\prime \prime}\right)$ or $C\left(\mathrm{VI}^{\prime \prime}\right)$ hold in $\mathrm{BCM}$ or not. An answer to these questions would refine the result above.

4.3. The embedding theorem. In this section we use Jech and Sochor's embedding theorem in an unorthodox way to obtain several results.

Given a set $X$, we define $\mathcal{P}^{\alpha}(X)$ for every ordinal $\alpha$ by recursion: $\mathcal{P}^{0}(X)$ $=X, \mathcal{P}^{\nu+1}(X)=\mathcal{P}\left(\mathcal{P}^{\nu}(X)\right)$, and $\mathcal{P}^{\lambda}(X)=\bigcup_{\nu<\lambda} \mathcal{P}^{\nu}(X)$. Now we state the embedding theorem:

Theorem 32 (Jech-Sochor). Let $\mathcal{U}$ be a model of ZFA $+\mathrm{AC}$, let $A$ be the set of atoms of $\mathcal{U}$, let $\mathcal{M}$ be the kernel (the class of pure sets) of $\mathcal{U}$, and let $\alpha$ be an ordinal in $\mathcal{U}$. Then, for every permutation model $\mathcal{V} \subset \mathcal{U}$ (a model of ZFA), there exists a symmetric extension $\mathcal{N} \supset \mathcal{M}$ (a model of $\mathrm{ZF})$ and a set $\widetilde{A} \in \mathcal{N}$ such that

$$
\left(\mathcal{P}^{\alpha}(A)\right)^{\mathcal{V}} \text { is } \in \text {-isomorphic to }\left(\mathcal{P}^{\alpha}(\widetilde{A})\right)^{\mathcal{N}} \text {. }
$$

For a full proof, refer to Jech [Jec73]. Here we will briefly describe the construction given there, and mention a property that is key for our purposes.

The construction can be described as follows: using forcing, we add to the kernel $\mathcal{M}$ (which is a model of ZFC) one generic set $\widetilde{a}$ of generic subsets of an ordinal $\kappa$ for each $a \in A$; the set $\widetilde{A}=\{\widetilde{a}: a \in A\}$ plays the role of the 
set of atoms. $\kappa$ is taken to be a regular cardinal such that $\kappa>\left|\mathcal{P}^{\alpha}(A)\right|$ (in $\mathcal{U}$ ), and the generic $\mathcal{M}[G]$ extension is obtained using forcing conditions of cardinality less than $\kappa$; this way it is guaranteed that the sets $\widetilde{a}$ do not appear in the first $\alpha$ levels of $\left(\mathcal{P}^{\alpha}(\widetilde{A})\right)^{\mathcal{M}[G]}$. Then $\mathcal{N}$ is obtained as a symmetric submodel of $\mathcal{M}[G]$ defined using suitable adaptations of the group and filter used to define $\mathcal{U}$.

The reason why sets of sets of ordinals are used, instead of simply using sets of ordinals, is that sets of ordinals have non-trivial definable relations between them; for example, any two sets of ordinals can be compared lexicographically. For the same reason, there should be no choice function for the set $\widetilde{A}$, otherwise the structure from the sets of ordinals would be lifted up to the elements of $\widetilde{A}$. This fact is stated in the following lemma:

LEMmA 33. Let $\mathcal{N}$ be the Jech-Sochor transfer of a permutation model $\mathcal{V}$, as defined above, with $\widetilde{A}$ being the set in correspondence with the set $A$ of atoms in $\mathcal{V}$. Then there is no infinite subset of $\widetilde{A}$ with a choice function.

There is no point in giving here a proof for this lemma without having a more detailed description of the construction of the embedding model. However, using the construction as presented in Jech [Jec73], it is not difficult to write a proof.

Theorem 32 is commonly used through a lemma like the following:

LEMMA 34. Let $\phi$ be a formula of the form $\exists \nu \psi(X, \nu)$, where the only quantifiers allowed in $\psi$ are of the form $\exists u \in \mathcal{P}^{\nu}(X)$ and $\forall u \in \mathcal{P}^{\nu}(X)$. If $\mathcal{V}$ is a permutation model such that $\mathcal{V} \vDash \exists X \phi(X)$, then there exists a symmetric model $\mathcal{N}$ of $\mathrm{ZF}$ such that $\mathcal{N} \vDash \exists X \phi(X)$.

A sentence like $\exists X \phi(X)$ is called boundable. Lemma 34 establishes that every boundable sentence is transferable, that is, if it is satisfied in a permutation model, there exists a Jech-Sochor symmetric model that also satisfies it. A few well known boundable sentences are the following:

1. $\exists X$ ( $X$ is amorphous).

2. $\exists X$ ( $X$ is an infinite II-finite set).

3. $\exists X$ ( $X$ is an infinite III-finite set).

4. $\exists X$ ( $X$ is an infinite IV-finite set).

5. $\exists X$ ( $X$ is an infinite $\Delta_{5}$-finite set).

The examples above, from 1 to 4, appear in Jech [Jec73]. For example 5, notice that if there is a surjection $f$ from a set $X$ onto $X \cup\{X\}$, and the rank of $X$ is $\gamma$, then the rank of $f$ is at most $\gamma+3$, and $f \in \mathcal{P}^{3}(X)$. Then the statement " $X$ is $\Delta_{5}$-finite" can be written as

$$
\begin{aligned}
\forall f \in \mathcal{P}^{3}(X) & (\exists y \in X \cup\{X\}, \forall x \in X(\langle x, y\rangle \notin f) \\
& \left.\vee\left(\exists y, y^{\prime} \in X \cup\{X\}, \exists x \in X\left(\langle x, y\rangle \in f \wedge\left\langle x, y^{\prime}\right\rangle \in f\right)\right)\right) .
\end{aligned}
$$


More general classes of formulas are transferable. In particular, so-called injectively and surjectively boundable statements are transferable; see Pincus [Pin72] for the definitions and a thorough discussion of the subject. We will not state those transfer theorems here for lack of space. However we will mention what results are being used, and the relevant references.

As a consequence of the previous lemmas, we obtain the following:

Theorem 35. In ZF:

1. $C$ (Ia) does not imply $C^{-}$(II),

2. $C\left(\Delta_{3}\right)$ does not imply $C^{-}(\mathrm{III})$,

3. $C$ (III) does not imply $C^{-}\left(\Delta_{3}\right)$,

4. $C(\mathrm{III})$ does not imply $C^{-}\left(\Delta_{5}\right)$,

5. $C(\mathrm{IV})$ does not imply $C^{-}(\mathrm{V})$.

Proof. All five parts will be proved in a similar way. In order to obtain a model of ZF where $C(\mathcal{Q})$ holds but $C^{-}\left(\mathcal{Q}^{\prime}\right)$ fails, we start with a permutation model $\mathcal{V}$ where $E(I, \mathcal{Q})$ holds (and consequently $C(\mathcal{Q})$ holds as well), while $A$ is an example of an infinite $\mathcal{Q}^{\prime}$-finite set. Then we take the Jech-Sochor transfer model $\mathcal{N}$; in this case the set $\widetilde{A}$ is an example of an infinite $\mathcal{Q}^{\prime}$-finite set without a partial choice function (by Lemma 33). The only detail is to show that $E(I, \mathcal{Q})$ still holds in $\mathcal{N}$.

For part 1, consider a permutation model referred to as Mathias-Pincus Model I in Howard and Rubin [HR98]. In the Jech-Sochor transfer of that model there are no amorphous sets (for the transferability of this statement, see Pincus [Pin72], 2B3), so $C$ (Ia) holds; however, $\widetilde{A}$ is a II-finite family without partial choice functions.

For part 2, consider Mostowski's ordered model. In the Jech-Sochor transfer of this model we find that every set is linearly orderable (for the transferability, see Pincus [Pin72], 4B4), so $E\left(\Delta_{3}, \mathrm{I}\right)$ and, consequently, $C\left(\Delta_{3}\right)$ hold. Nevertheless, $\widetilde{A}$ is a III-finite family without a partial choice function.

For parts 3 and 4, take the Second Fraenkel Model. That model satisfies $E(\mathrm{I}, \mathrm{III})$, and this sentence transfers because it is surjectively boundable. Therefore $C$ (III) holds in $\mathcal{N}$. Still, $\widetilde{A}$ is a $\Delta_{3^{-}}$and $\Delta_{5}$-finite family without a partial choice function.

Finally, for part 5, consider the modified Fraenkel model obtained from a model of ZFA +AC with uncountably many atoms using the group of all permutations of the set of atoms and countable supports. This model satisfies $E(\mathrm{I}, \mathrm{IV})$, and this sentence transfers since it is injectively bounded. Therefore, $C(\mathrm{IV})$ holds in the Jech-Sochor transfer of the model. Of course, $\widetilde{A}$ is a V-finite family without a partial choice function. 


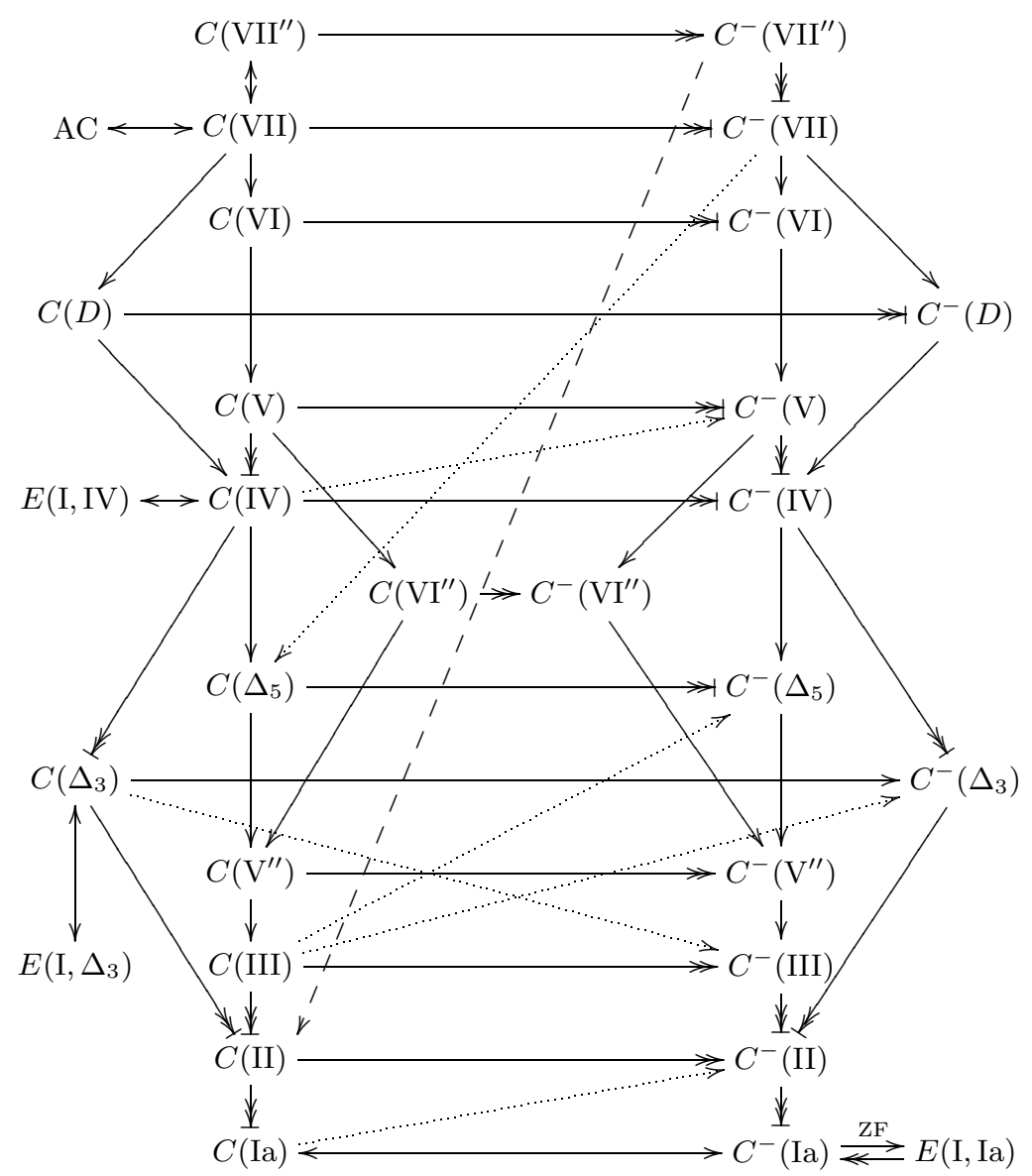

Fig. 2. Implications between principles. Solid arrows represent implications in ZFA. Double headed arrows cannot be reversed in ZFA. Arrows with two heads and a bar cannot be reversed in ZF. The dashed arrow represents an implication that cannot be proved in ZFA. Dotted arrows represent implications that cannot be proved in ZF.

All the independence results in this section are summarized in Figure 2. Relations not specified in Figure 2 are open questions; of particular interest are the following: Does $C^{-}(\mathrm{VII})$ (or $\left.C^{-}\left(\mathrm{VII}^{\prime \prime}\right)\right)$ imply $C\left(\mathrm{~V}^{\prime \prime}\right)$ (or $C\left(\Delta_{3}\right)$ or $\left.C\left(\mathrm{VI}^{\prime \prime}\right)\right)$ ?

\section{References}

[Bla77] A. Blass, Ramsey's theorem in the hierarchy of choice principles, J. Symbolic Logic 42 (1977), 387-390.

[HR98] P. Howard and J. E. Rubin, Consequences of the Axiom of Choice, Amer. Math. Soc., Providence, RI, 1998. 
[HS] P. Howard and L. Spišiak, Definitions of finite and the power set operation, preprint.

[HY89] P. E. Howard and M. F. Yorke, Definitions of finite, Fund. Math. 133 (1989), 169-177.

[Jec73] T. J. Jech, The Axiom of Choice, Stud. Logic Found. Math. 75, North-Holland, Amsterdam, 1973.

[Lév58] A. Lévy, The independence of various definitions of finiteness, Fund. Math. 46 (1958), 1-13.

[Lév65] - The Fraenkel-Mostowski method for independence proofs in set theory, in: Theory of Models (Berkeley, 1963), North-Holland, Amsterdam, 1965, 221-228.

[Mos38] A. Mostowski, Über den Begriff einer endlichen Menge, C. R. Séances Soc. Sci. Lettres Varsovie 31 (1938), 13-20.

[Pin72] D. Pincus, Zermelo-Fraenkel consistency results by Fraenkel-Mostowski methods, J. Symbolic Logic 37 (1972), 721-743.

[Spi93] L. Spišiak, Dependences between definitions of finiteness. II, Czechoslovak Math. J. 43 (118) (1993), 391-407.

[SV88] L. Spišiak and P. Vojtáš, Dependences between definitions of finiteness, ibid. 38 (113) (1988), 389-397.

[Tar24] A. Tarski, Sur les ensembles finis, Fund. Math. 6 (1924), 45-95.

[Tar38] -, Ein Überdeckungssatz für endliche Mengen nebst einigen Bemerkungen über die Definitionen der Endlichkeit, ibid. 30 (1938), 156-163.

[Tru74] J. Truss, Classes of Dedekind finite cardinals, ibid. 84 (1974), 187-208.

Department of Mathematics

Purdue University

West Lafayette, IN 47907-1395, U.S.A.

E-mail: odlc@math.purdue.edu

Received 14 May 2001;

in revised form 15 December 2001 